KOBE-TH-02-04

hep-th/0212079

\title{
Comments on Quiver Gauge Theories and Matrix Models
}

\author{
Shigenori Seki ${ }^{\dagger}$ \\ Department of Physics, Faculty of Science \\ Kobe University, Kobe 65\%-8501, Japan
}

\begin{abstract}
Dijkgraaf and Vafa have conjectured the correspondences between topological string theories, $\mathcal{N}=1$ gauge theories and matrix models. By the use of this conjecture, we calculate the quantum deformations of Calabi-Yau threefolds with ADE singularities from ADE multi-matrix models. We obtain the effective superpotentials of the dual quiver gauge theories in terms of the geometric engineering for the deformed geometries. We find the Veneziano-Yankielowicz terms in the effective superpotentials.
\end{abstract}

December 2002

$\dagger$ sekis@phys.sci.kobe-u.ac.jp 


\section{Introduction}

String theories give us a lot of useful methods in order for us to understand various gauge theories. For example, the AdS/CFT correspondence [1] and the gauge/gravity correspondence [2] are known well. A-model topological string theories correspond to Chern-Simons gauge theories by the gauge/gravity correspondence.

There are mirror symmetry between the A-model topological string theories and the B-model topological string theories. Recently Dijkgraaf and Vafa have proposed the correspondences between the B-model topological string theories, $\mathcal{N}=1$ supersymmetric gauge theories and large $N$ matrix models [3 5 ]. In other words, these correspondences are the mirror dual of the gauge/gravity correspondence. The $\mathcal{N}=1$ gauge theory is constructed by adding certain superpotential to the $\mathcal{N}=2$ gauge theory. In the description of D-brane configuration, the $\mathcal{N}=1$ gauge theory is realized by D5-branes wrapped on two-cycles in Calabi-Yau manifolds. When the two-cycles are blown down, new three-cycles emerge. Three-form RR and NSNS fluxes then appear instead of the D5-branes. This is called geometric transition [6 [10]. On the Calabi-Yau manifolds after the geometric transition, there are two kinds of three cycles, which are compact $A_{i}$-cycles and non-compact $B_{i}$-cycles. We define periods,

$$
\mu_{i}=\frac{1}{2 \pi i} \oint_{A_{i}} \Omega, \quad \Pi_{i}=\frac{\partial \mathcal{F}_{0}}{\partial \mu_{i}}=\int_{B_{i}} \Omega,
$$

where $\Omega$ is a holomorphic three-form on the Calabi-Yau threefold and $\mathcal{F}_{0}$ is a prepotential. In terms of these periods we can write down the effective superpotential of the dual gauge theory,

$$
W_{\mathrm{eff}}=\sum_{i}\left(N_{i} \Pi_{i}-2 \pi i \tau_{i} \mu_{i}\right),
$$

where $N_{i}$ is a number of D-branes and $\tau_{i}$ is a gauge coupling.

It is proposed that the effective superpotential can be reproduced by the matrix model with certain tree level superpotential $W(\Phi)$ [3]. The partition function of the matrix model is $Z=\int d \Phi \exp \left(-\frac{1}{g_{s}} W(\Phi)\right)$. Fixing $S_{i}=N_{i} g_{s}$, we take the limit $N_{i} \gg 1, g_{s} \ll 1$, and the partition function then leads to $Z=\exp \sum_{g} g_{s}^{2 g-2} \mathcal{F}_{g}\left(S_{i}\right)$. The free energies $\mathcal{F}_{g}$ are the contributions of genus $g$ diagrams. In particular $\mathcal{F}_{0}$ is derived from the planar diagrams. If we can calculate the partition function, we obtain the free energy, $\log Z$. We can identify it with the prepotential of the dual gauge theory and we obtain the exact effective superpotential in terms of (1.1) and (1.2). Since the perturbative analyses in the 
matrix models lead to the exact results in the dual gauge theories, this new derivation of the superpotentials is powerful. A lot of works on this subject have been done [11] 50].

In this paper we consider $\mathcal{N}=1$ quiver gauge theories and matrix models. The matrix models are ADE multi-matrix models, which have been studied in [51,52]. The quiver gauge theories are realized by the string theories on the Calabi-Yau manifolds with ADE singularities. The $\mathcal{N}=2$ quiver gauge theories lead to the $\mathcal{N}=1$ theories by the additional superpotentials, while the dual Calabi-Yau geometries are deformed. These deformations are reproduced by the matrix model [4]. Since we systematically introduce a lot of gauge symmetries to the quiver gauge theories, they are interesting also for realistic particle theories [23].

In Section 2, we will analyse the quantum deformations of the ADE singularities in the matrix model side. In terms of the deformed geometries, we will calculate the superpotentials of the dual quiver gauge theories. Section 3 is devoted to the conclusions and the some comments on left problems.

\section{Effective superpotentials of quiver gauge theories}

Before discussing on the quiver gauge theories and the multi-matrix models, we will give a brief review on a one-matrix model [3] . Let us consider an $N \times N$ Hermitean matrix $\Phi$. The partition function of the one-matrix model with the tree level superpotential $W(\Phi)$ is

$$
Z=\int d \Phi \exp \left(-\frac{1}{g_{s}} W(\Phi)\right)
$$

where we set that $W(\Phi)$ is a degree $n$ polynomial of $\Phi$. In terms of the $N$ eigen values $\lambda_{I}$ $(I=1, \cdots, N)$ of $\Phi$, we can rewrite the partition function (2.1) as

$$
Z=\int\left(\prod_{I=1}^{N} d \lambda_{I}\right) \Delta(\lambda)^{2} \exp \left(-\frac{1}{g_{s}} \sum_{I=1}^{N} W\left(\lambda_{I}\right)\right)
$$

where $\Delta(\lambda)$ is the Vandermonde determinant, $\prod_{I<J}\left(\lambda_{I}-\lambda_{J}\right)$. When we describe the partition function as $Z=\int \Pi_{I} d \lambda_{I} e^{-\hat{S}}$, the effective action $\hat{S}$ is denoted by

$$
\hat{S}=\frac{1}{g_{s}} \sum_{I=1}^{N} W\left(\lambda_{I}\right)-2 \sum_{I<J} \log \left(\lambda_{I}-\lambda_{J}\right) .
$$


From the action (2.3), the equations of motion for $\lambda_{I}$ are written down as

$$
\frac{1}{g_{s}} W^{\prime}\left(\lambda_{I}\right)-2 \sum_{J \neq I} \frac{1}{\lambda_{I}-\lambda_{J}}=0 .
$$

We introduce a resolvent,

$$
\omega(x) \equiv \frac{1}{N} \sum_{I=1}^{N} \frac{1}{x-\lambda_{I}},
$$

which is useful in the matrix model technology [53,54]. Physical meaning of the resolvent is a loop operator and we can easily derive a loop equation from (2.4) in terms of the resolvent. From (2.4), we define the function,

$$
y(x)=W^{\prime}(x)-2 g_{s} \sum_{J=1}^{N} \frac{1}{x-\lambda_{J}}=W^{\prime}(x)-2 S \omega(x),
$$

where $S$ is the 't Hooft coupling $N g_{s} \cdot \omega(x)$ is not a polynomial, but, in large $N$ limit, $y(x)^{2}$ is given by $y(x)^{2}=W^{\prime}(x)^{2}+f_{n-1}(x)$, where $f_{n-1}(x)$ is a degree $n-1$ polynomial.

In the context of large $N$ duality and geometric transitions [6 9], the dual Calabi-Yau geometry after the deformation is denoted by

$$
u^{2}+v^{2}+y^{2}+W^{\prime}(x)^{2}+f_{n-1}(x)=0 .
$$

We then consider the one-form

$$
y(x) d x=\sqrt{W^{\prime}(x)^{2}+f_{n-1}(x)} d x .
$$

The periods (1.1) are described as

$$
\frac{1}{2 \pi i} \int_{A_{i}} y(x) d x=\mu_{i}, \quad \int_{B_{i}}^{\Lambda^{\frac{3}{2}}} y(x) d x=\frac{\partial \mathcal{F}_{0}}{\partial \mu_{i}}=\Pi_{i},
$$

in terms of the one-form (2.8). Without the deformation, $y(x)$ in (2.8) is equal to $W^{\prime}(x)$. Since the function (2.6) derived from the matrix model can be identified with $y(x)$ in (2.8), $-2 S \omega(x)$ in (2.6) leads to $f_{n-1}(x)$ in (2.7), in other words, $f_{n-1}(x)$ is regarded as the contribution of loop operators in the matrix model. Adding $f_{n-1}$ is called a quantum deformation. 
Let us now consider the ADE singularities and the quiver gauge theories. The CalabiYau threefolds with the ADE singularities are realized by the fibration of two dimensional ADE singularities. The fibres over $x$-plane are denoted by

$$
\begin{array}{ll}
A_{n} \quad u^{2}+v^{2}+\prod_{i=1}^{n+1}\left(y-t_{i}(x)\right)=0, \quad \sum_{i=1}^{n+1} t_{i}=0 \\
D_{n} \quad u^{2}+v^{2} y+\frac{1}{y}\left(\prod_{i=1}^{n}\left(y-t_{i}(x)^{2}\right)-\prod_{i=1}^{n} t_{i}(x)^{2}\right)+2 \prod_{i=1}^{n} v t_{i}(x)=0 \\
E_{6} \quad u^{2}+v^{3}+y^{4}+\epsilon_{2}(x) v y^{2}+\epsilon_{5}(x) v y+\epsilon_{6}(x) y^{2}+\epsilon_{8}(x) v \\
\quad+\epsilon_{9}(x) y+\epsilon_{12}(x)=0 \\
E_{7} \quad u^{2}+v^{3}+v y^{3}+\epsilon_{2}(x) v^{2} y+\epsilon_{6}(x) v^{2}+\epsilon_{8}(x) v y+\epsilon_{10}(x) y^{2} \\
\quad+\epsilon_{12}(x) v+\epsilon_{14}(x) y+\epsilon_{18}(x)=0 \\
E_{8} \quad u^{2}+v^{3}+y^{5}+\epsilon_{2}(x) v y^{3}+\epsilon_{8}(x) v y^{2}+\epsilon_{12}(x) y^{3}+\epsilon_{14}(x) v y \\
& +\epsilon_{18}(x) y^{2}+\epsilon_{20}(x) v+\epsilon_{24}(x) y+\epsilon_{30}(x)=0
\end{array}
$$

where $\epsilon_{i}$ are functions of $t_{i}(x)$ and are explicitly written down in [55]. For these fibrations we can describe the one-forms $y_{i}(x) d x$ [8] as

$$
\begin{aligned}
& A_{n} \quad y_{i}=t_{i+1}-t_{i}, \quad i=1, \cdots, n, \\
& D_{n} \quad y_{i}=t_{i+1}-t_{i}, \quad i=1, \cdots, n-1, \quad y_{n}=-t_{n-1}-t_{n}, \\
& E_{n} \quad y_{i}=t_{i+1}-t_{i}, \quad i=1, \cdots, n-1, \quad y_{n}=t_{1}+t_{2}+t_{3} \text {. }
\end{aligned}
$$

If we calculate the periods (2.9) for the one-forms (2.15), (2.16) and (2.17), we obtain the effective superpotentials of the quiver gauge theories.

In the following, we will derive the quantum deformations of the ADE singularities from the ADE matrix models and consider the effective superpotentials of the dual quiver gauge theories.

\section{1. $A_{n}$ singularity}

Firstly we consider the $A_{n}$ singularities and the $A_{n}$ quiver gauge theories.

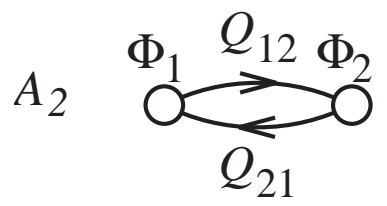

fig. $1 A_{2}$ quiver diagram 
For the simplest example, let us study an $A_{2}$ singularity [4]. The $A_{2}$ quiver diagram is similar to the $A_{2}$ Dynkin diagram and is represented in fig. 1. We assign a $U\left(N_{i}\right)$ gauge group to the $i$-th node of the $A_{2}$ diagram. The dual quiver gauge theory consists of the adjoint scalars $\Phi_{1}, \Phi_{2}$ and the bifundamental matters $Q_{12}, Q_{21}$ transforming in the representation of $\left(N_{1}, \bar{N}_{2}\right),\left(N_{2}, \bar{N}_{1}\right)$ respectively. The tree level superpotential is

$$
W(\Phi, Q)=\operatorname{Tr} Q_{12} \Phi_{2} Q_{21}-\operatorname{Tr} Q_{21} \Phi_{1} Q_{12}+W_{1}\left(\Phi_{1}\right)+W_{2}\left(\Phi_{2}\right)
$$

The supersymmetry of the quiver gauge theory is broken from $\mathcal{N}=2$ to $\mathcal{N}=1$ by inserting the superpotentials $W_{i}\left(\Phi_{i}\right)$. The partition function of the matrix model which we should consider is $Z=\int d \Phi d Q \exp \left(-\frac{1}{g_{s}} W(\Phi, Q)\right) . \Phi_{i}$ is an $N_{i} \times N_{i}$ matrix and $Q_{i j}$ is an $N_{i} \times N_{j}$ matrix. Integrating $Q_{i j}$ out in the partition function, we obtain the effective action of $\Phi_{i}$. Since $\Phi_{i}$ is the $N_{i} \times N_{i}$ matrix, using the eigen values $\lambda_{i, I}\left(i=1,2, \quad I=1, \cdots, N_{i}\right)$, we exchange the matrix integrals $\int d \Phi$ for the eigen value integrals $\int d \lambda_{i, I}$. We then obtain the equations of motion [四,

$$
\begin{aligned}
& \frac{1}{g_{s}} W_{1}^{\prime}\left(\lambda_{1, I}\right)-2 \sum_{J=1, J \neq I}^{N_{1}} \frac{1}{\lambda_{1, I}-\lambda_{1, J}}+\sum_{J=1}^{N_{2}} \frac{1}{\lambda_{1, I}-\lambda_{2, J}}=0, \\
& \frac{1}{g_{s}} W_{2}^{\prime}\left(\lambda_{2, I}\right)-2 \sum_{J=1, J \neq I}^{N_{2}} \frac{1}{\lambda_{2, I}-\lambda_{2, J}}+\sum_{J=1}^{N_{1}} \frac{1}{\lambda_{2, I}-\lambda_{1, J}}=0 .
\end{aligned}
$$

The second terms in (2.18) and (2.19) are the contributions of loop effects of $\lambda_{i, I}$, and the third terms come from the contributions of $Q_{i j}$. On the analogy of (2.5), we define resolvents,

$$
\omega_{1}(x) \equiv \frac{1}{N_{1}} \sum_{I=1}^{N_{1}} \frac{1}{x-\lambda_{1, I}}, \quad \omega_{2}(x) \equiv \frac{1}{N_{2}} \sum_{I=1}^{N_{2}} \frac{1}{x-\lambda_{2, I}} .
$$

By the way, the classical deformation of the $A_{2}$ singularity is denoted by

$$
u^{2}+v^{2}+\left(y-t_{1}^{\mathrm{cl}}(x)\right)\left(y-t_{2}^{\mathrm{cl}}(x)\right)\left(y-t_{3}^{\mathrm{cl}}(x)\right)=0, \quad \sum_{i=1}^{3} t_{i}^{\mathrm{cl}}(x)=0,
$$

where the deformation parameters $t_{i}^{\mathrm{cl}}(x)$ are given by

$$
t_{1}^{\mathrm{cl}}(x)=-\frac{2 W_{1}^{\prime}(x)+W_{2}^{\prime}(x)}{3}, \quad t_{2}^{\mathrm{cl}}(x)=\frac{W_{1}^{\prime}(x)-W_{2}^{\prime}(x)}{3}, \quad t_{3}^{\mathrm{cl}}(x)=\frac{W_{1}^{\prime}(x)+2 W_{2}^{\prime}(x)}{3},
$$


from (2.10) and (2.15) [8]. On the other hand, from (2.18), (2.19) and (2.20), we obtain the one-form $y_{i}(x) d x$ which are described as

$$
y_{1}(x)=W_{1}^{\prime}(x)-2 S_{1} \omega_{1}(x)+S_{2} \omega_{2}(x), \quad y_{2}(x)=W_{2}^{\prime}(x)+S_{1} \omega_{1}(x)-2 S_{2} \omega_{2}(x) .
$$

Since the classical deformations are given by $y_{1}^{\mathrm{cl}}(x)=t_{2}^{\mathrm{cl}}-t_{1}^{\mathrm{cl}}=W_{1}^{\prime}(x)$ and $y_{2}^{\mathrm{cl}}(x)=$ $t_{3}^{\mathrm{cl}}-t_{2}^{\mathrm{cl}}=W_{2}^{\prime}(x)$, the terms including $\omega_{i}$ in (2.23) imply quantum effects. We can define the quantum deformation of the $A_{2}$ singularity [4] as

$$
u^{2}+v^{2}+\left(y-t_{1}(x)\right)\left(y-t_{2}(x)\right)\left(y-t_{3}(x)\right)=0, \quad \sum_{i=1}^{3} t_{i}(x)=0,
$$

so that $t_{i}(x)$ satisfy $y_{1}(x)=t_{2}(x)-t_{1}(x)$ and $y_{2}(x)=t_{3}(x)-t_{2}(x)$. Actually $t_{i}(x)$ can be written down as

$t_{1}(x)=t_{1}^{\mathrm{cl}}(x)+S_{1} \omega_{1}(x), \quad t_{2}(x)=t_{2}^{\mathrm{cl}}(x)-S_{1} \omega_{1}(x)+S_{2} \omega_{2}(x), \quad t_{3}(x)=t_{3}^{\mathrm{cl}}(x)-S_{2} \omega_{2}(x)$.

So far we have given a brief review on the geometry of the $A_{2}$ quiver $[4]$. We will generalize the above discussions to the $A_{n}$ quiver and calculate the effective superpotentials of $A_{n}$ quiver gauge theories. We will consider, in particular, the quadratic tree level superpotentials.

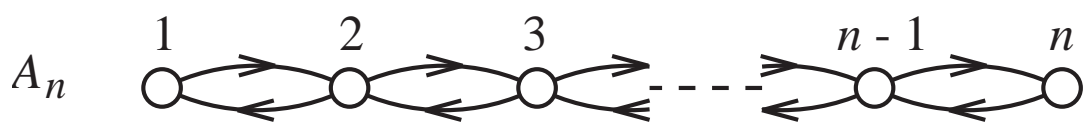

fig. $2 A_{n}$ quiver diagram

The $A_{n}$ quiver diagram is represented in fig. 2. Since the diagram has $n$ nodes, we assign a $U\left(N_{i}\right)$ gauge group to each $i$-th node. The dual theory is the $A_{n}$ quiver gauge theory which consists of the adjoint scalars $\Phi_{i}$ and the bifundamental matters $Q_{i, i+1}, Q_{i+1, i}$. The tree level superpotential is

$$
W(\Phi, Q)=\sum_{i=1}^{n-1} \operatorname{Tr}\left(Q_{i, i+1} \Phi_{i+1} Q_{i+1, i}-Q_{i+1, i} \Phi_{i} Q_{i, i+1}\right)+\sum_{i=1}^{n} \operatorname{Tr} W_{i}\left(\Phi_{i}\right),
$$

where $W_{i}\left(\Phi_{i}\right)$ is a polynomial of $\Phi_{i}$. We integrate out $Q_{i j}$ in the partition function,

$$
Z=\int \prod_{i=1}^{n} d \Phi_{i} \prod_{i, j} d Q_{i j} \exp \left(-\frac{1}{g_{s}} W(\Phi, Q)\right)
$$


and we can rewrite it in terms of the eigen values $\lambda_{i, I}\left(I=1, \cdots, N_{i}\right)$ of $\Phi_{i}$. We then obtain

$$
\begin{aligned}
Z & =\int \prod_{i=1}^{n} d \Phi_{i} \frac{1}{\prod_{i} \operatorname{det}\left(\Phi_{i} \otimes \mathbf{1}-\mathbf{1} \otimes \Phi_{i+1}\right)} \exp \left(-\frac{1}{g_{s}} W(\Phi, Q)\right) \\
& =\int \prod_{i, I} d \lambda_{i, I} \frac{\prod_{i, I<J}\left(\lambda_{i, I}-\lambda_{i, J}\right)^{2}}{\prod_{i, I, J}\left(\lambda_{i, I}-\lambda_{i+1, J}\right)} \exp \left(-\frac{1}{g_{s}} \sum_{i, I} W_{i}\left(\lambda_{i, I}\right)\right) .
\end{aligned}
$$

When we describe the partition function as $\int \prod_{i, I} d \lambda_{i, I} e^{-\hat{S}}$, the effective action $\hat{S}$ is denoted by

$$
\hat{S}=\frac{1}{g_{s}} \sum_{i, I} W_{i}\left(\lambda_{i, I}\right)-2 \sum_{i, I<J} \log \left(\lambda_{i, I}-\lambda_{i, J}\right)+\sum_{i, I, J} \log \left(\lambda_{i, I}-\lambda_{i+1, J}\right) .
$$

The equations of motion for the action (2.27) become

$$
\begin{aligned}
& \frac{1}{g_{s}} W_{1}^{\prime}\left(\lambda_{1, I}\right)-2 \sum_{J=1, J \neq I}^{N_{1}} \frac{1}{\lambda_{1, I}-\lambda_{1, J}}+\sum_{J=1}^{N_{2}} \frac{1}{\lambda_{1, I}-\lambda_{2, J}}=0, \\
& \frac{1}{g_{s}} W_{j}^{\prime}\left(\lambda_{j, I}\right)-2 \sum_{J=1, J \neq I}^{N_{j}} \frac{1}{\lambda_{j, I}-\lambda_{j, J}} \\
& +\sum_{J=1}^{N_{j-1}} \frac{1}{\lambda_{j, I}-\lambda_{j-1, J}}+\sum_{J=1}^{N_{j+1}} \frac{1}{\lambda_{j, I}-\lambda_{j+1, J}}=0, \quad j=2, \cdots, n-1, \\
& \frac{1}{g_{s}} W_{n}^{\prime}\left(\lambda_{n, I}\right)-2 \sum_{J=1, J \neq I}^{N_{n}} \frac{1}{\lambda_{n, I}-\lambda_{n, J}}+\sum_{J=1}^{N_{n-1}} \frac{1}{\lambda_{n, I}-\lambda_{n-1, J}}=0 .
\end{aligned}
$$

We also introduce the resolvents,

$$
\omega_{i}(x) \equiv \frac{1}{N_{i}} \sum_{I=1}^{N_{i}} \frac{1}{x-\lambda_{i, I}}, \quad i=1, \cdots, n
$$

and the 't Hooft couplings $S_{i} \equiv g_{s} N_{i}$. Note that, in the context of the large $N$ duality, we fix $S_{i}$ and take the limits where $N_{i}$ go to infinity. We then read the following functions from $(2.28)$ as

$$
\begin{aligned}
& y_{1}(x)=W_{1}^{\prime}(x)-2 S_{1} \omega_{1}(x)+S_{2} \omega_{2}(x) \\
& y_{j}(x)=W_{j}^{\prime}(x)-2 S_{j} \omega_{j}(x)+S_{j-1} \omega_{j-1}(x)+S_{j+1} \omega_{j+1}(x), \quad j=2, \cdots, n-1, \\
& y_{n}(x)=W_{n}^{\prime}(x)-2 S_{n} \omega_{n}(x)+S_{n-1} \omega_{n-1}(x) .
\end{aligned}
$$


$y_{i}(x)$ include the quantum deformations of $A_{n}$ singularity and $y_{i}(x) d x$ denote the deformed one-forms. Note that $y_{i}^{\mathrm{cl}}=W_{i}^{\prime}$ are regarded as the classical deformations.

Let us set the tree level superpotentials to be the quadratic ones,

$$
W_{i}(x)=\frac{m_{i}}{2} x^{2}, \quad i=1, \cdots, n .
$$

From $W_{i}^{\prime}\left(\lambda_{i, I}\right)=0$, the classical vacua are $\lambda_{i, I}=0$. Since the perturbative analyses around these vacua in the matrix model give us the exact effective superpotentials of the $\mathcal{N}=1$ dual gauge theories, we approximate $\lambda_{i, I}$ to the vacuum expectation values, that is, we set all $\lambda_{i, I}$ to be equal to zero. (2.30) then becomes

$$
y_{1}(x)=m_{1} x-\frac{2 S_{1}-S_{2}}{x}=\frac{m_{1}}{x}\left(x-a_{1}^{+}\right)\left(x-a_{1}^{-}\right),
$$

where $a_{1}^{ \pm}= \pm \sqrt{\frac{2 S_{1}-S_{2}}{m_{1}}}$. The critical point $a_{1}=0$ of $W_{1}^{\prime}\left(a_{1}\right)=0$ is splitted to the two points $a_{1}^{ \pm}$. In the same way, each critical point $a_{j}=0(j=2, \cdots, n-1)$ is splitted to the points $a_{j}^{ \pm}= \pm \sqrt{\frac{2 S_{j}-S_{j-1}-S_{j+1}}{m_{j}}}$, and $a_{n}=0$ is splitted to $a_{n}^{ \pm}= \pm \sqrt{\frac{2 S_{n}-S_{n-1}}{m_{n}}}$. In other words, every original critical point is resolved to the two points. By the use of these resolved points, the periods (2.9) are described as

$$
\mu_{i}=\frac{1}{2 \pi i} \int_{a_{i}^{-}}^{a_{i}^{+}} y_{i}(x) d x, \quad \Pi_{i}=\int_{a_{i}^{+}}^{\Lambda_{i}^{\frac{3}{2}}} y_{i}(x) d x .
$$

Since the $B_{i}$-cycles are non-compact, the cut-off $\Lambda_{i}$ are needed. From (2.30), (2.31) and (2.32), we obtain the periods around the B-cycles,

$$
\begin{aligned}
\Pi_{1}= & \frac{1}{2} m_{1} \Lambda_{1}^{3}-\frac{1}{2}\left(2 S_{1}-S_{2}\right)\left(1-\log \frac{2 S_{1}-S_{2}}{\Lambda_{1}^{3}}\right)-\frac{1}{2}\left(2 S_{1}-S_{2}\right) \log m_{1}, \\
\Pi_{j}= & \frac{1}{2} m_{j} \Lambda_{j}^{3}-\frac{1}{2}\left(2 S_{j}-S_{j-1}-S_{j+1}\right)\left(1-\log \frac{2 S_{j}-S_{j-1}-S_{j+1}}{\Lambda_{j}^{3}}\right) \\
& -\frac{1}{2}\left(2 S_{j}-S_{j-1}-S_{j+1}\right) \log m_{j}, \quad j=2, \cdots, n-1, \\
\Pi_{n}= & \frac{1}{2} m_{n} \Lambda_{n}^{3}-\frac{1}{2}\left(2 S_{n}-S_{n-1}\right)\left(1-\log \frac{2 S_{n}-S_{n-1}}{\Lambda_{n}^{3}}\right) \\
& -\frac{1}{2}\left(2 S_{n}-S_{n-1}\right) \log m_{n} .
\end{aligned}
$$

We also calculate the periods around the A-cycles as

$$
\begin{aligned}
\mu_{1} & =\frac{1}{2}\left(2 S_{1}-S_{2}\right), \\
\mu_{j} & =\frac{1}{2}\left(2 S_{j}-S_{j-1}-S_{j+1}\right), \quad j=2, \cdots, n-1, \\
\mu_{n} & =\frac{1}{2}\left(2 S_{n}-S_{n-1}\right) .
\end{aligned}
$$


Using these results and (1.2), we obtain the effective superpotential,

$$
\begin{aligned}
W_{\text {eff }}= & \sum_{i=1}^{n} \frac{1}{2} N_{i} m_{i} \Lambda_{i}^{3} \\
& -\frac{1}{2}\left[N_{1}\left(2 S_{1}-S_{2}\right)\left(1-\log \frac{2 S_{1}-S_{2}}{\Lambda_{1}^{3}}\right)+N_{n}\left(2 S_{n}-S_{n-1}\right)\left(1-\log \frac{2 S_{n}-S_{n-1}}{\Lambda_{n}^{3}}\right)\right. \\
& \left.+\sum_{i=2}^{n-1} N_{i}\left(2 S_{i}-S_{i-1}-S_{i+1}\right)\left(1-\log \frac{2 S_{i}-S_{i-1}-S_{i+1}}{\Lambda_{i}^{3}}\right)\right] \\
& -\frac{1}{2}\left[N_{1}\left(2 S_{1}-S_{2}\right) \log m_{1}+N_{n}\left(2 S_{n}-S_{n-1}\right) \log m_{n}\right. \\
& \left.+\sum_{i=2}^{n-1} N_{i}\left(2 S_{i}-S_{i-1}-S_{i+1}\right) \log m_{i}\right] \\
& +\pi i\left[\tau_{1}\left(2 S_{1}-S_{2}\right)+\tau_{n}\left(2 S_{n}-S_{n-1}\right)+\sum_{i=2}^{n-1} \tau_{i}\left(2 S_{i}-S_{i-1}-S_{i+1}\right)\right]
\end{aligned}
$$

We can reproduce the Veneziano-Yankielowicz terms [56], which appear in the second term of (2.41). Note that, if we set all $N_{i}=N$, all $m_{i}=1$, all $\Lambda_{i}=\Lambda$, all $S_{i}=S$ and all $\tau_{i}=\tau$, the effective superpotential is denoted simply by

$$
W_{\text {eff }}=\frac{1}{2} n N \Lambda^{3}-N S\left(1-\log \frac{S}{\Lambda^{3}}\right)+2 \pi i \tau S .
$$

Note that the constant terms $\sum_{i=1}^{n} \frac{1}{2} N_{i} m_{i} \Lambda_{i}^{3}$ in (2.41) and $\frac{1}{2} n N \Lambda^{3}$ in (2.42) can be ignored.

\section{2. $D_{n}$ singularity}

Next let us consider the $D_{n}$ singularities. A $D_{4}$ singularity appears in the compactifications of F-theory and is discussed also in the context of Dijkgraaf-Vafa conjecture 23.

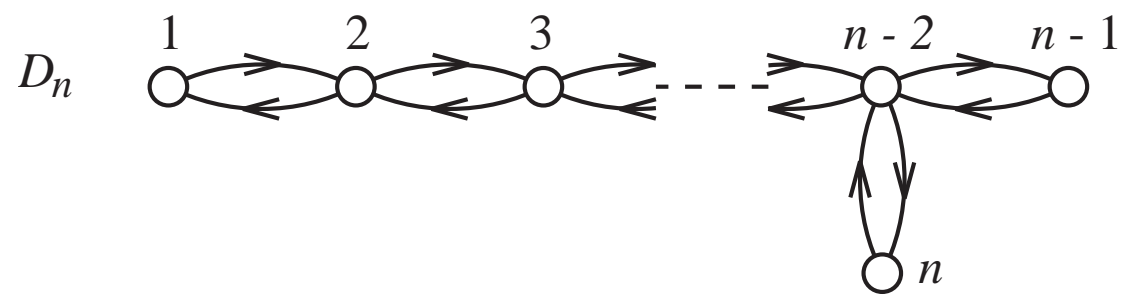

fig. $3 D_{n}$ quiver diagram 
The $D_{n}$ quiver diagram is represented in fig. 3 . In the same way as the $A_{n}$ quiver gauge theories, we assign a $U\left(N_{i}\right)$ gauge group to each $i$-th node. The fields defined in the $D_{n}$ quiver gauge theories are the adjoint scalars $\Phi_{i}$ for $i=1, \cdots, n$ and the bifundamental matters $Q_{i j}$ for the $i$-th and $j$-th nodes which are linked to each other.

The tree level superpotential is

$$
\begin{aligned}
W(\Phi, Q)= & \sum_{i=1}^{n-1} \operatorname{Tr}\left(Q_{i, i+1} \Phi_{i+1} Q_{i+1, i}-Q_{i+1, i} \Phi_{i} Q_{i, i+1}\right) \\
& +\operatorname{Tr}\left(Q_{n-2, n} \Phi_{n} Q_{n, n-2}-Q_{n, n-2} \Phi_{n-2} Q_{n-2, n}\right)+\sum_{i=1}^{n} \operatorname{Tr} W_{i}\left(\Phi_{i}\right)
\end{aligned}
$$

Integrating $Q_{i j}$ out and rewriting the matrix integrals of $\Phi_{i}$ with the eigen value integrals of $\lambda_{i, I}$ which are the eigen values of $\Phi_{i}$, we obtain the equations of motion for $\lambda_{i, I}$. From these equations of motion, we find the one-forms $y_{i}(x) d x$ of the deformed $D_{n}$ singularity,

$$
\begin{aligned}
y_{1}(x)= & W_{1}^{\prime}(x)-2 S_{1} \omega_{1}(x)+S_{2} \omega_{2}(x) \\
y_{j}(x)= & W_{j}^{\prime}(x)-2 S_{j} \omega_{j}(x)+S_{j-1} \omega_{j-1}(x)+S_{j+1} \omega_{j+1}(x), \quad j=2, \cdots, n-3,(x) \\
y_{n-2}(x)= & W_{n-2}^{\prime}(x)-2 S_{n-2} \omega_{n-2}(x)+S_{n-3} \omega_{n-3}(x)+S_{n-1} \omega_{n-1}(x) \\
& +S_{n} \omega_{n}(x) \\
y_{n-1}(x)= & W_{n-1}^{\prime}(x)-2 S_{n-1} \omega_{n-1}(x)+S_{n-2} \omega_{n-2}(x) \\
y_{n}(x)= & W_{n}^{\prime}(x)-2 S_{n} \omega_{n}(x)+S_{n-2} \omega_{n-2}(x)
\end{aligned}
$$

by the use of the resolvents which are defined in the same way as (2.29). In the $D_{n}$ case different from the $A_{n}$ case, (2.46) is characteristic, because the $(n-2)$-th node is linked to the three nodes. The one-forms $y_{i}(x) d x$ include the quantum effects coming from the $D_{n}$ matrix models.

Let us consider the quadratic superpotential (2.33). We can then calculate the periods around the B-cycles as

$$
\begin{aligned}
\Pi_{1}= & \frac{1}{2} m_{1} \Lambda_{1}^{3}-\frac{1}{2}\left(2 S_{1}-S_{2}\right)\left(1-\log \frac{2 S_{1}-S_{2}}{\Lambda_{1}^{3}}\right)-\frac{1}{2}\left(2 S_{1}-S_{2}\right) \log m_{1}, \\
\Pi_{j}= & \frac{1}{2} m_{j} \Lambda_{j}^{3}-\frac{1}{2}\left(2 S_{j}-S_{j-1}-S_{j+1}\right)\left(1-\log \frac{2 S_{j}-S_{j-1}-S_{j+1}}{\Lambda_{j}^{3}}\right) \\
& -\frac{1}{2}\left(2 S_{j}-S_{j-1}-S_{j+1}\right) \log m_{j}, \quad j=2, \cdots, n-3,
\end{aligned}
$$




$$
\begin{aligned}
\Pi_{n-2}= & \frac{1}{2} m_{n-2} \Lambda_{n-2}^{3}-\frac{1}{2}\left(2 S_{n-2}-S_{n-3}-S_{n-1}-S_{n}\right)\left(1-\log \frac{2 S_{n-2}-S_{n-3}-S_{n-1}-S_{n}}{\Lambda_{n-2}^{3}}\right) \\
& -\frac{1}{2}\left(2 S_{n-2}-S_{n-3}-S_{n-1}-S_{n}\right) \log m_{n-2} \\
\Pi_{n-1}= & \frac{1}{2} m_{n-1} \Lambda_{n-1}^{3}-\frac{1}{2}\left(2 S_{n-1}-S_{n-2}\right)\left(1-\log \frac{2 S_{n-1}-S_{n-2}}{\Lambda_{n-1}^{3}}\right) \\
& -\frac{1}{2}\left(2 S_{n-1}-S_{n-2}\right) \log m_{n-1} \\
\Pi_{n}= & \frac{1}{2} m_{n} \Lambda_{n}^{3}-\frac{1}{2}\left(2 S_{n}-S_{n-2}\right)\left(1-\log \frac{2 S_{n}-S_{n-2}}{\Lambda_{n}^{3}}\right)-\frac{1}{2}\left(2 S_{n}-S_{n-2}\right) \log m_{n}
\end{aligned}
$$

and the periods around the A-cycles as

$$
\begin{aligned}
\mu_{1} & =\frac{1}{2}\left(2 S_{1}-S_{2}\right), \\
\mu_{j} & =\frac{1}{2}\left(2 S_{j}-S_{j-1}-S_{j+1}\right), \quad j=2, \cdots, n-3, \\
\mu_{n-2} & =\frac{1}{2}\left(2 S_{n-2}-S_{n-3}-S_{n-1}-S_{n}\right), \\
\mu_{n-1} & =\frac{1}{2}\left(2 S_{n-1}-S_{n-2}\right), \\
\mu_{n} & =\frac{1}{2}\left(2 S_{n}-S_{n-2}\right) .
\end{aligned}
$$

From these periods, we can obtain the effective superpotentials in terms of (1.2).

For example, we consider the $D_{4}$ quiver gauge theory. The effective superpotential becomes

$$
\begin{aligned}
W_{\mathrm{eff}}= & -\frac{1}{2} N\left(2 S_{1}-S_{2}\right)\left(1-\log \frac{2 S_{1}-S_{2}}{\Lambda^{3}}\right)-\frac{1}{2} N\left(2 S_{3}-S_{2}\right)\left(1-\log \frac{2 S_{3}-S_{2}}{\Lambda^{3}}\right) \\
& -\frac{1}{2} N\left(2 S_{4}-S_{2}\right)\left(1-\log \frac{2 S_{4}-S_{2}}{\Lambda^{3}}\right) \\
& -\frac{1}{2} N\left(2 S_{2}-S_{1}-S_{3}-S_{4}\right)\left(1-\log \frac{2 S_{2}-2 S_{1}-S_{3}-S_{4}}{\Lambda^{3}}\right) \\
& +\pi i \tau\left(S_{1}+S_{3}+S_{4}-S_{2}\right),
\end{aligned}
$$

where, for simplicity, we set that all $m_{i}=1$, all $N_{i}=N$, all $\Lambda_{i}=\Lambda$ and all $\tau_{i}=\tau$, and the constant term $\sum_{i} \frac{1}{2} N_{i} m_{i} \Lambda_{i}^{3}$ is ignored. Since the first, third and fourth nodes of the $D_{4}$ quiver diagram have a cyclic symmetry, $S_{1}, S_{3}, S_{4}$ in the superpotential (2.59) can be replaced with one another. 


\section{3. $E_{n}$ singularity}

Finally we consider the $E_{n}$ singularities. In the string theories, the $E_{n}$ singularities play important roles. For example, $E_{8} \times E_{8}$ gauge symmetry of heterotic string theories are realized by the $E_{n}$ singular fibres in the F-theory. So it is interesting to analyse the $E_{n}$ singularities.

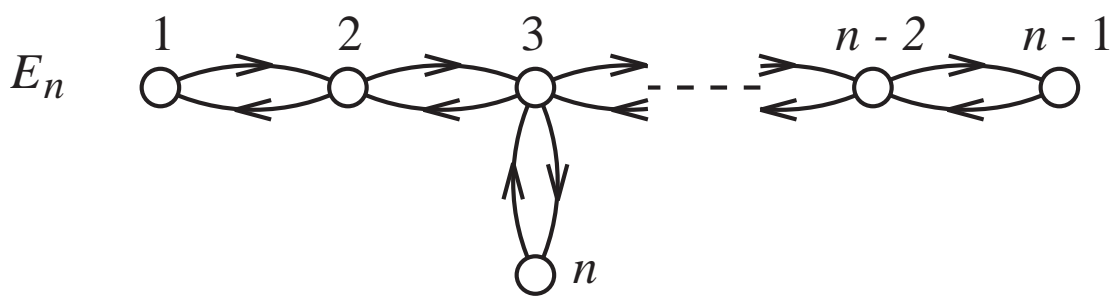

fig. $4 E_{n}$ quiver diagram

The $E_{n}$ quiver diagram is depicted in fig. 4 . Each $i$-th node has a $U\left(N_{i}\right)$ gauge group. In the same way as the $A_{n}$ and $D_{n}$ quiver gauge theories, we define the adjoint scalars $\Phi_{i}$ and the bifundamental matters $Q_{i j}$. The tree level superpotential in the $E_{n}$ quiver matrix models is

$$
\begin{aligned}
W(\Phi, Q)= & \sum_{i=1}^{n-1} \operatorname{Tr}\left(Q_{i, i+1} \Phi_{i+1} Q_{i+1, i}-Q_{i+1, i} \Phi_{i} Q_{i, i+1}\right) \\
& +\operatorname{Tr}\left(Q_{3 n} \Phi_{n} Q_{n 3}-Q_{n 3} \Phi_{3} Q_{3 n}\right)+\sum_{i=1}^{n} \operatorname{Tr} W_{i}\left(\Phi_{i}\right) .
\end{aligned}
$$

We integrate $Q_{i j}$ out in the partition function $Z=\int d \Phi d Q \exp \left(-\frac{1}{g_{s}} W(\Phi, Q)\right)$ and obtain the effective action of $\lambda_{i, I}$ which are the eigen values of $\Phi_{i}$. We calculate the equations of motion from this effective action and read the following functions,

$$
\begin{aligned}
y_{1}(x)= & W_{1}^{\prime}(x)-2 S_{1} \omega_{1}(x)+S_{2} \omega_{2}(x), \\
y_{2}(x)= & W_{2}^{\prime}(x)-2 S_{2} \omega_{2}(x)+S_{1} \omega_{1}(x)+S_{3} \omega_{3}(x), \\
y_{3}(x)= & W_{3}^{\prime}(x)-2 S_{3} \omega_{3}(x)+S_{2} \omega_{2}(x)+S_{4} \omega_{4}(x)+S_{n} \omega_{n}(x), \\
y_{j}(x)= & W_{j}^{\prime}(x)-2 S_{j} \omega_{j}(x)+S_{j-1} \omega_{j-1}(x) \\
& +S_{j+1} \omega_{j+1}(x), \quad j=4, \cdots, n-2, \\
y_{n-1}(x)= & W_{n-1}^{\prime}(x)-2 S_{n-1} \omega_{n-1}(x)+S_{n-2} \omega_{n-2}(x), \\
y_{n}(x)= & W_{n}^{\prime}(x)-2 S_{n} \omega_{n}(x)+S_{3} \omega_{3}(x),
\end{aligned}
$$


We also consider the quadratic superpotential (2.33) and assume the eigen values in $\omega_{i}(x)$ to be in the vacua, that is, $\lambda_{i, I}=0$. Since every critical point of $W_{i}(x)$ is then splitted to the two points by the quantum deformations, we calculate the period (2.34) for the one-form $y_{i}(x) d x$. The periods $\Pi_{i}$ around the B-cycles are

$$
\begin{aligned}
& \Pi_{1}=\frac{1}{2} m_{1} \Lambda_{1}^{3}-\frac{1}{2}\left(2 S_{1}-S_{2}\right)\left(1-\log \frac{2 S_{1}-S_{2}}{\Lambda_{1}^{3}}\right)-\frac{1}{2}\left(2 S_{1}-S_{2}\right) \log m_{1}, \\
& \Pi_{2}=\frac{1}{2} m_{2} \Lambda_{2}^{3}-\frac{1}{2}\left(2 S_{2}-S_{1}-S_{3}\right)\left(1-\log \frac{2 S_{2}-S_{1}-S_{3}}{\Lambda_{2}^{3}}\right) \\
& -\frac{1}{2}\left(2 S_{2}-S_{1}-S_{3}\right) \log m_{2} \\
& \Pi_{3}=\frac{1}{2} m_{3} \Lambda_{3}^{3}-\frac{1}{2}\left(2 S_{3}-S_{2}-S_{4}-S_{n}\right)\left(1-\log \frac{2 S_{3}-S_{2}-S_{4}-S_{n}}{\Lambda_{3}^{3}}\right) \\
& -\frac{1}{2}\left(2 S_{3}-S_{2}-S_{4}-S_{n}\right) \log m_{3}, \\
& \Pi_{j}=\frac{1}{2} m_{j} \Lambda_{j}^{3}-\frac{1}{2}\left(2 S_{j}-S_{j-1}-S_{j+1}\right)\left(1-\log \frac{2 S_{j}-S_{j-1}-S_{j+1}}{\Lambda_{j}^{3}}\right) \\
& -\frac{1}{2}\left(2 S_{j}-S_{j-1}-S_{j+1}\right) \log m_{j}, \quad j=4, \cdots, n-2, \\
& \Pi_{n-1}=\frac{1}{2} m_{n-1} \Lambda_{n-1}^{3}-\frac{1}{2}\left(2 S_{n-1}-S_{n-2}\right)\left(1-\log \frac{2 S_{n-1}-S_{n-2}}{\Lambda_{n-1}^{3}}\right) \\
& -\frac{1}{2}\left(2 S_{n-1}-S_{n-2}\right) \log m_{n-1}, \\
& \Pi_{n}=\frac{1}{2} m_{n} \Lambda_{n}^{3}-\frac{1}{2}\left(2 S_{n}-S_{3}\right)\left(1-\log \frac{2 S_{n}-S_{3}}{\Lambda_{n}^{3}}\right)-\frac{1}{2}\left(2 S_{n}-S_{3}\right) \log m_{n} .
\end{aligned}
$$

and the periods $\mu_{i}$ around the A-cycles are

$$
\begin{aligned}
\mu_{1} & =\frac{1}{2}\left(2 S_{1}-S_{2}\right), \\
\mu_{2} & =\frac{1}{2}\left(2 S_{2}-S_{1}-S_{3}\right), \\
\mu_{3} & =\frac{1}{2}\left(2 S_{3}-S_{2}-S_{4}-S_{n}\right), \\
\mu_{j} & =\frac{1}{2}\left(2 S_{j}-S_{j-1}-S_{j+1}\right), \quad j=4, \cdots, n-2, \\
\mu_{n-1} & =\frac{1}{2}\left(2 S_{n-1}-S_{n-2}\right), \\
\mu_{n} & =\frac{1}{2}\left(2 S_{n}-S_{3}\right) .
\end{aligned}
$$

From these periods and (1.2), we can calculate the effective superpotentials of the $E_{n}$ quiver gauge theories. For simplicity, we ignore the terms independent of $S_{i}$ and set that 
all $m_{i}=1$, all $N_{i}=N$, all $\Lambda_{i}=\Lambda$ and all $\tau_{i}=\tau$. For example the effective superpotential of the $E_{8}$ quiver then becomes

$$
\begin{aligned}
W_{\mathrm{eff}}= & -\frac{1}{2} N\left(2 S_{1}-S_{2}\right)\left(1-\log \frac{2 S_{1}-S_{2}}{\Lambda^{3}}\right)-\frac{1}{2} N\left(2 S_{7}-S_{6}\right)\left(1-\log \frac{2 S_{7}-S_{6}}{\Lambda^{3}}\right) \\
& -\frac{1}{2} N\left(2 S_{8}-S_{3}\right)\left(1-\log \frac{2 S_{8}-S_{3}}{\Lambda^{3}}\right) \\
& -\frac{1}{2} N\left(2 S_{3}-S_{2}-S_{4}-S_{8}\right)\left(1-\log \frac{2 S_{3}-S_{2}-S_{4}-S_{8}}{\Lambda^{3}}\right) \\
& -\frac{1}{2} N \sum_{j=2,4,5,6}\left(2 S_{j}-S_{j-1}-S_{j+1}\right)\left(1-\log \frac{2 S_{j}-S_{j-1}-S_{j+1}}{\Lambda^{3}}\right) \\
& +\pi i \tau\left(S_{1}+S_{7}+S_{8}-S_{3}\right) .
\end{aligned}
$$

In this effective superpotential we can also find Veniziano-Yankielowicz terms. Since the third node is linked to the three nodes, $S_{3}$ is characteristic in the $E_{n}$ quiver as well as in the $D_{n}$ quiver.

\section{Conclusions and discussions}

We have considered the Calabi-Yau manifolds with the ADE singularities. If we calculate the periods of one-forms $y_{i}(x) d x$ around compact A-cycles and non-compact Bcycles on the deformed geometry of the Calabi-Yau manifolds, we can obtain the effective superpotentials of the dual gauge theories by the geometric engineering. We have calculated the equations of motion in the ADE multi-matrix models. Since the quantum deformations are derived from the perturbative analyses of the matrix models by the Dijkgraaf-Vafa conjecture, We have found out the quantum deformations of the one-forms $y_{i}(x) d x$ from those equations of motion in the ADE matrix models.

We have considered the quadratic superpotentials $W_{i}\left(\Phi_{i}\right)=\frac{1}{2} m_{i} \operatorname{Tr} \Phi_{i}^{2}$ for the simple examples. Then the classical vacua are $\lambda_{i, I}=0$, where $\lambda_{i, I}$ are the eigen values of $\Phi_{i}$. Since the perturbation theory on these vacua gives rise to the effective superpotential in the dual gauge theory, we have approximated $\lambda_{i, I}$ in the resolvents $\omega_{i}$ to the vacua. The original critical point $a_{i}=0$, which is obtained from $\left(y_{i}^{\mathrm{cl}}=\right) W_{i}^{\prime}\left(a_{i}\right)=0$, is splitted to the two points $a_{i}^{ \pm}$, which are derived from $y_{i}\left(a_{i}^{ \pm}\right)=0$ on the deformed geometry. In terms of $a_{i}^{ \pm}$and the cut-off parameters $\Lambda_{i}$, we have calculated the periods (2.34). From these periods we have written down the effective superpotentials of the dual quiver gauge theories. We have also found that the effective superpotentials include the Veneziano-Yankielowicz terms. 
We have used the approximation, that is, all eigen values of $\Phi_{i}$ appearing in the resolvents are in the classical vacua. But in order to derive exact effective superpotentials for the $\mathcal{N}=1$ quiver gauge theories, we should achieve the integration of the eigen values in the multi-matrix model partition function.

Though it is difficult to exactly calculate the partition functions of the multi-matrix models, we can analyse the loop expansions of the planar diagrams order by order of the 't Hooft couplings $S_{i}$ by the use of Feynman diagrams [19]. So we should confirm the expansions in the context of the geometric engineering.

\section{Acknowledgement}

I would like to thank B. Taylor for useful comments. This work is supported in part by the Grant-in-Aid for Scientific Research of Professor H. Sonoda, Kobe Univ., from Japan Ministry of Education, Science and Culture (\#14340077). 


\section{References}

[1] J. Maldacena, "The Large $N$ Limit of Superconformal Field Theories and Supergravity", Adv. Theor. Math. Phys. 2 (1998) 231; Int. J. Theor. Phys. 38 (1999) 1113, hep-th/9711200.

[2] R. Gopakumar and C. Vafa, "On the Gauge Theory/Geometry Correspondence", Adv. Theor. Math. Phys. 3 (1999) 1415, hep-th/9811131.

[3] R. Dijkgraaf and C. Vafa, "Matrix Models, Topological Strings, and Supersymmetric Gauge Theories", Nucl. Phys. B644 (2002) 3, hep-th/0206255.

[4] R. Dijkgraaf and C. Vafa, "On Geometry and Matrix Models", Nucl. Phys. B644 (2002) 21, hep-th/0207106.

[5] R. Dijkgraaf and C. Vafa, "A Perturbative Window into Non-Perturbative Physics", hep-th/0208048.

[6] C. Vafa, "Superstrings and Topological Strings at Large N", J. Math. Phys. 42 (2001) 2798, hep-th/0008142.

[7] F. Cachazo, K. Intriligator and C. Vafa, "A Large $N$ Duality via a Geometric Transition", Nucl. Phys. B603 (2001) 3, hep-th/0103067.

[8] F. Cachazo, K. Intriligator and C. Vafa, "Geometric Transitions and $N=1$ Quiver Theories", hep-th/0108120.

[9] F. Cachazo, B. Fiol, K. Intriligator, S. Katz and C. Vafa, "A Geometric Unification of Dualities", Nucl. Phys. B628 (2002) 3, hep-th/0110028.

[10] K. Oh and R. Tatar, "Duality and Confinement in $\mathcal{N}=1$ Supersymmetric Theories from Geometric Transitions", Adv. Theor. Math. Phys. 6 (2002) 141, hepth/0112040.

[11] L. Chekhov and A. Mironov, "Matrix Models vs. Seiberg-Witten/Whitham Theories", hep-th/0209085.

[12] N. Dorey, T. J. Hollowood, S. P. Kumar and A. Sinkovics, "Exact Superpotentials from Matrix Models", hep-th/0209089.

[13] N. Dorey, T. J. Hollowood, S. P. Kumar and A. Sinkovics, "Massive Vacua of $\mathcal{N}=1$ " Theory and S-duality from Matrix Models", hep-th/0209099.

[14] M. Aganagic and C. Vafa, "Perturbative Derivation of Mirror Symmetry", hepth/0209138.

[15] G. Bonelli, "Matrix String Models for Exact (2,2) String Theories in R-R Backgrounds", hep-th/0209225.

[16] F. Ferrari, "On Exact Superpotentials in Confining Vacua", hep-th/0210135.

[17] H. Fuji and Y. Ookouchi, "Comments on Effective Superpotentials via Matrix Models", hep-th/0210148.

[18] D. Berenstein, "Quantum Moduli Spaces from Matrix Models", hep-th/0210183. 
[19] R. Dijkgraaf, S. Gukov, V. A. Kazakov and C. Vafa, "Perturbative Analysis of Gauged Matrix Models", hep-th/0210238.

[20] N. Dorey, T. J. Hollowood and S. P. Kumar, "S-duality of the Leigh-Strassler Deformation via Matrix Models", hep-th/0210239.

[21] A. Gorsky, "Konishi Anomaly and N=1 Effective Superpotentials from Matrix Models", hep-th/0210281.

[22] R. Argurio, V. L. Campos, G. Ferretti and R. Heise, "Exact Superpotentials for Theories with Flavors via a Matrix Integral", hep-th/0210291.

[23] J. McGreevy, "Adding Flavor to Dijkgraaf-Vafa", hep-th/0211009.

[24] R. Dijkgraaf, M. T. Grisaru, C. S. Lam, C. Vafa and D. Zanon, "Perturbative Computation of Glueball Superpotentials", hep-th/0211017.

[25] H. Suzuki, "Perturbative Derivation of Exact Superpotential for Meson Fields from Matrix Theories with One Flavour", hep-th/0211052.

[26] F. Ferrari, "Quantum Parameter Space and Double Scaling Limits in $\mathcal{N}=1$ Super Yang-Mills Theory", hep-th/0211069.

[27] I. Bena and R. Roiban, "Exact Superpotentials in $N=1$ Theories with Flavor and their Matrix Model Formulation", hep-th/0211075.

[28] Y. Demasure and R. A. Janik, "Effective Matter Superpotentials from Wishart Random Matrices", hep-th/0211082.

[29] M. Aganagic, A. Klemm, M. Marino and C. Vafa, "Matrix Model as a Mirror of Chern-Simons Theory", hep-th/0211098.

[30] R. Gopakumar, "N $=1$ Theories and a Geometric Master Field", hep-th/0211100.

[31] S. Naculich, H. Schnitzer and N. Wyllard, "The $\mathcal{N}=2 U(N)$ Gauge Theory Prepotential and Periods from a Perturbative Matrix Model Calculation", hep-th/0211123.

[32] F. Cachazo, M. R. Douglas, N. Seiberg and E. Witten, "Chiral Rings and Anomalies in Supersymmetric Gauge Theory", hep-th/0211170.

[33] Y. Tachikawa, "Derivation of the Konishi anomaly relation from Dijkgraaf-Vafa with (Bi-)fundamental matter", hep-th/0211189.

[34] R. Dijkgraaf, A. Neitzke and C. Vafa, "Large N Strong Coupling Dynamics in NonSupersymmetric Orbifold Field Theories", hep-th/0211194.

[35] B. Feng, "Seiberg Duality in Matrix Model", hep-th/0211202.

[36] A. Klemm, M. Marino and S. Theisen, "Gravitational Corrections in Supersymmetric Gauge Theory and Matrix Models", hep-th/0211216.

[37] B. Feng and Y.-H. He, "Seiberg Duality in Matrix Models II", hep-th/0211234.

[38] V. A. Kazakov and A. Marshakov, "Complex Curve of the Two Matrix Model and its Tau-function", hep-th/0211236.

[39] R. Dijkgraaf, A. Sinkovics and M. Temurhan, "Matrix Models and Gravitational Corrections", hep-th/0211241. 
[40] H. Itoyama and A. Morozov, "The Dijkgraaf-Vafa Prepotential in the Context of General Seiberg-Witten Theory", hep-th/0211245.

[41] R. Argurio, V. L. Campos, G. Ferretti and R. Heise, "Baryonic Corrections to Superpotentials from Perturbation Theory", hep-th/0211249.

[42] S. Naculich, H. Schnitzer and N. Wyllard, "Matrix Model Approach to the $\mathcal{N}=$ $2 U(N)$ Gauge Theory with Matter in the Fundamental Representation", hepth/0211254.

[43] H. Itoyama and A. Morozov, "Experiments with the WDVV Equations for the Gluinocondensate Prepotential: the Cubic (two-cut) Case", hep-th/0211259.

[44] H. Ita, H. Nieder and Y. Oz, "Perturbative Computation of Glueball Superpotentials for $\mathrm{SO}(\mathrm{N})$ and $\mathrm{USp}(\mathrm{N})$ ", hep-th/0211261.

[45] I. Bena, R. Roiban and R. Tatar, "Baryons, Boundaries and Matrix Models", hepth/0211271.

[46] Y. Ookouchi, "N $=1$ Gauge Theory with Flavor from Fluxes", hep-th/0211287.

[47] S. K. Ashok, R. Corrado, N. Halmagyi, K. D. Kennaway and C. Romelsberger, "Unoriented Strings, Loop Equations, and $\mathcal{N}=1$ Superpotentials from Matrix Models", hep-th/0211291.

[48] K. Ohta, "Exact Mesonic Vacua from Matrix Models", hep-th/0212025.

[49] H. Itoyama and A. Morozov, "Calculating Gluino-Condensate Prepotential", hepth/0212032.

[50] R. A. Janik and N. A. Obers, " $S O(N)$ Superpotential, Seiberg-Witten Curves and Loop Equations", hep-th/0212069.

[51] S. Kharchev, A. Marshakov, A. Mironov, A. Morozov and S. Pakuliak, "Conformal Matrix Models as an Alternative to Conventional Multi-Matrix Models", Nucl. Phys. B404 (1993) 717, hep-th/9208044.

[52] I. Kostov, "Gauge Invariant Matrix Model for the Â-D̂-Ê Closed Strings", Phys. Lett. B297 (1992) 74, hep-th/9208053.

[53] P. Ginsparg and G. Moore, "Lectures on 2D Gravity and 2D String Theory", TASI 1992, hep-th/9304011.

[54] P. Di Francesco, P. Ginsparg and J. Zinn-Justin, "2D Gravity and Random Matrices", Phys. Rept. 254 (1995) 1, hep-th/9306153.

[55] S. Katz and D. R. Morrison, "Gorenstein Threefold Singularities with Small Resolutions via Invariant Theory for Weyl Groups", J. Alg. Geom. 1 (1992) 449, alggeom/9202002.

[56] G. Veneziano and S. Yankielowicz, "An Effective Lagrangian for the Pure $\mathcal{N}=1$ Supersymmetric Yang-Mills Theory", Phys. Lett. B113 (1982) 231. 